\title{
Inferring Signed Networks from Preschoolers' Observed Parallel and Social Play
}

\author{
Jennifer Watling Neal, Zachary P. Neal, C. Emily Durbin \\ Michigan State University
}

\begin{abstract}
Early childhood is an important developmental period for network formation. However, the observational methods used for measuring young children's networks present challenges for capturing both positive and negative ties. To overcome these challenges, we explored the use of a bipartite projection backbone model for inferring both negative and positive ties from observational data of children's play. Using observational data collected in one 3-year-old $(\mathrm{N}=17)$ and one 4-year-old $(\mathrm{N}=18)$ preschool classroom, we examined whether patterns of homophily, triadic closure, and balance in networks inferred using this method matched theoretical and empirical expectations from the early childhood literature. Consistent with this literature, we found that signed networks inferred using a backbone model exhibited gender homophily in positive ties and gender heterophily in negative ties. Additionally, networks inferred from social play exhibited more closed and balanced triads than networks inferred from parallel play. These findings offer evidence of the validity of bipartite projection backbone models for inferring signed networks from preschoolers' observed play.
\end{abstract}

Keywords: signed networks, backbone model, preschool, children, play

\section{Introduction}

Preschool offers an initial opportunity for young children to form both positive and negative social ties with same-age peers (e.g., Daniel et al., 2016; Irwin et al., 2021; Martin et al., 2005; Schaefer et al., 2010). However, studies of networks in early childhood are rare (Neal, 2020) and only a handful of these studies have examined signed networks that include both positive and negative ties (e.g., Daniel et al., 2016; Van den Oord et al., 2000). Because self-report methods are difficult to use with young children, researchers have often measured early childhood networks using scan observational methods that focus on affiliation behaviors such as play (e.g., DeLay et al., 2016; Neal, 2020; Schaefer et al., 2010; Strayer \& Santos, 1996). These observational methods present challenges for capturing both positive and negative ties between children.

Email addresses: jneal@msu.edu (Jennifer Watling Neal), zpneal@msu.edu (Zachary P. Neal), cdurbin@msu.edu (C. Emily Durbin)

Preprint submitted to PsyArXiv
It is not always clear how many times a pair of children need to be observed playing together to warrant concluding that the pair have a positive tie. Additionally, overt negative peer interactions such as conflict are low rate and more subtle negative peer interactions like avoidance are difficult to directly observe in young children.

To overcome these challenges, we explored the use of a bipartite projection backbone model for inferring both negative and positive ties from observational data of children's play. We found that patterns of homophily, triadic closure, and balance in networks inferred using this method matched theoretical and empirical expectations from the early childhood literature. Specifically, consistent with gender schema theory and gendered patterns of play (e.g., Martin \& Halverson, 1981; Martin \& Ruble, 2004; Martin et al., 2013; Daniel et al., 2016; Van den Oord et al., 2000), our inferred signed networks exhibited gender homophily in positive ties and gender heterophily in negative ties. Additionally, consistent with theoretical expectations about levels of coordination required for different types of

February 15, 2022 
play (e.g., Parten, 1932; Coplan \& Arbeau, 2009), networks inferred from social play exhibited more closed and balanced triads than networks inferred from parallel play. These findings offer evidence of the validity of bipartite projection backbone models for inferring signed networks from preschoolers' observed play.

We begin by providing a brief review of the literature on networks in early childhood, including literature on measurement challenges as well as theoretical and empirical expectations for patterns of homophily, triadic closure, and balance. Next, we describe the use of one bipartite projection backbone model - the fixed degree sequence model for inferring signed networks from observations of children's parallel and social play. Using observational data collected in two preschool classrooms, we offer evidence for the validity of this method by demonstrating that patterns of homophily, triadic closure, and balance are consistent with theoretical expectations based on gender and type of play. Finally, we end with implications for understanding networks in early childhood and future directions for research.

\section{Background}

Early childhood is an important developmental period for the formation of both positive and negative ties (Daniel et al., 2016). In the U.S. and in other countries, it is common for young children to enroll in preschool, providing one of the first opportunities for extended social interactions with other same-age peers (e.g., Daniel et al., 2016; Irwin et al., 2021; Martin et al., 2005; Schaefer et al., 2010). During this period, young children begin to engage in more complex and coordinated forms of play (Coplan \& Arbeau, 2009; Göncü et al., 2002) and form networks with standard structural features like reciprocity and transitivity (Daniel et al., 2013, 2016; Schaefer et al., 2010). These networks can influence children's personality traits (e.g., Neal et al., 2017), preschool competency (e.g., DeLay et al., 2016), and engagement in gender stereotypical activities (e.g., Martin et al., 2013).

Although early childhood is an important developmental period, fewer network studies have been conducted during this period than in middle childhood or adolescence. In a recent systematic review of developmental psychology articles using networks, only $4 \%$ included samples ages 0 to 4
(Neal, 2020). Researchers may be less likely to examine networks in early childhood because the selfreport name generators typically used to collect network data (see adams, 2020) are developmentally challenging to use with young children. Unlike older children and adolescents, young children typically require extra supports when completing these selfreport name generators including pictures of all of the children within the specified network boundary (e.g., class, school). Children either sort or point to these pictures in response to the self-report name generator (e.g., Daniel et al., 2016; McCandless \& Marshall, 1957; Van den Oord et al., 2000). In the United States, self-report methods may also not be feasible when measuring negative ties because educators, parents, and university institutional review boards are often reluctant to allow researchers to use name generators focused on negative ties with young children.

Because self-report name generators are difficult to use with young children, researchers have typically used more time-intensive scan observational methods to measure networks in early childhood (e.g., Daniel et al., 2019; DeLay et al., 2016; Hanish et al., 2005; Neal et al., 2017; Schaefer et al., 2010). However, there are three major challenges to using observational approaches to measure young children's networks. First, it is not always clear how many times two children must be observed interacting to warrant inferring they have a positive network tie. Schaefer et al. (2010), Daniel et al. (2013, 2019), and Neal et al. (2017) have all proposed solutions, but none offer a formal statistical test. Second, in preschool, directly observable negative interactions such as overt displays of negative affect, physical aggression, or conflict often occur at low rates and are disrupted by teachers (Roseth et al., 2008; Vaughn et al., 2003). Observing children for longer periods of time can help (e.g., Ostrov \& Keating, 2004), but is even more time and labor intensive. Third, more subtle negative ties such as disliking or avoidance are challenging to directly observe because they often do not involve overt behaviors.

In this paper, we explored bipartite projection backbone models as a possible solution to these challenges (Neal, 2014). The data collected via scan observational methods can be organized as a binary child-by-play event matrix $\mathbf{M}$, where $M_{i k}=1$ if child $i$ was observed participating in a play event $k$, and thus can be viewed as a two-mode or bipartite network. When applied to these data, backbone 
models offer a formal statistical testing framework for inferring, on the basis of observed co-playing, whether two children have a positive network tie. Moreover, they can also be used to infer, on the basis of observations of not playing together, whether two children are avoiding one another and thus have a negative network tie.

In this study, we were interested in whether a network inferred from scan observational data using a backbone model validly measures the network among preschool children. One way to provide evidence of the validity of a measurement approach involves examining whether the measurement yields theoretically and empirically expected patterns (Cronbach \& Meehl, 1955; Messick, 1995). To generate this type of evidence of validity, here we examine whether the inferred inferred network exhibits structural patterns that are theoretically expected and have previously been empirically observed among preschool children. In the remainder of this section, we review patterns rooted in gender- and play-differences that offer concrete expectations, and thus which can be used to evaluate the validity of the network inferences.

\subsection{Gender and Play in Early Childhood}

Gender structures children's social ties from an early age. By preschool, children exhibit strong preferences for same gender peers and networks of positive ties exhibit notable gender homophily (e.g., Chen et al., 2020; Daniel et al., 2016; Martin et al., 2005, 2013; Schaefer et al., 2010; Van den Oord et al., 2000). In contrast, negative ties in early childhood tend to exhibit gender heterophily. Preschool children are more likely to indicate that they dislike playing with different-gender peers, and this dislike increases between ages 3 and 5 (Daniel et al., 2016; Van den Oord et al., 2000).

Gender patterns in networks during early childhood are driven by multiple mechanisms. First, children may exhibit direct preferences for playing with same gender peers (Maccoby, 1990; Maccoby \& Jacklin, 1987; Martin \& Ruble, 2010; Martin et al., 2011). These preferences can be explained by gender schema theory which suggests that young children make assumptions about the characteristics of boys and girls and view samegender peers as more similar to themselves (Martin \& Halverson, 1981; Martin \& Ruble, 2004). Second, boys and girls may prefer to participate in distinct gender-typed activities during play (e.g., playing with trucks vs. dolls), which might indirectly lead to gender homophily in positive ties and gender heterophily in negative ties (Goble et al., 2012; La Freniere et al., 1984; Martin \& Fabes, 2001; Martin et al., 2011, 2013).

We can offer evidence of the validity of backbone models for inferring negative and positive ties in preschoolers' observed play by examining whether gender patterns conform to established theories and prior empirical findings. If backbone models validly infer young children's networks, positive ties derived from these models should more often occur between same gender peers and negative ties derived from these models should more often occur between peers of different genders.

\subsection{Types of Play in Early Childhood}

During preschool, children commonly participate in multiple forms of play including parallel play and social play (Coplan \& Arbeau, 2009; Luckey \& Fabes, 2005; Parten, 1932). Parallel play occurs when a child passively participates in the same activities as a nearby peer or set of peers but does not engage in conversation or interactions with them. For example, two children who are silently building their own block towers next to each other would be engaged in parallel play. In contrast, social play occurs when a child actively participates in direct interactions such as social conversations, sharing toys, or pretend play with a peer or set of peers. For example, two children who are working together to build the same block tower by sharing blocks and discussing what to build next would be engaged in social play.

Networks reflecting parallel play and social play may differ in structure. As Parten (1932) noted parallel play involves "playing beside rather than with the other children" (p. 250). By definition, parallel play does not involve any direct social interactions with peers, and therefore patterns of coparallel play may be driven more by opportunity than by coordination. In contrast, social play is characterized by social interactions that are often complex and involve coordination between peers (e.g., sharing, division of labor; Coplan \& Arbeau, 2009). Because social play involves coordination between peers, networks derived from social play observations should contain more structures that suggest coordination, while networks derived from parallel play observations should contain fewer such structures.

One network structure that can require coordination to form is triadic closure. In a dynamic con- 
text, triadic closure occurs when open triads of the form A-B-C (what Granovetter (1973) calls 'forbidden triads') become closed triads because a network tie forms between $\mathrm{A}$ and $\mathrm{C}$. There are several mechanisms that might induce triadic closure, including brokerage (e.g., B introduces A to C), homophily (e.g., A and $\mathrm{C}$ have similar interests, namely B), and co-presence (e.g., A and $\mathrm{C}$ are in the same place at the same time when they are with B). The effects of triadic closure are reflected in static networks when a large fraction of connected triples are also triangles (i.e. high transitivity). While triad closure is common in most networks, it is also known to be common and to increase over time specifically among preschool children (e.g., liking, social play; Daniel et al., 2016, 2019; Schaefer et al., 2010; Van den Oord et al., 2000). Because social play involves more coordination than parallel play, if backbone models validly infer young children's networks, we would expect networks inferred from social play to exhibit more transitivity than networks inferred from parallel play.

A second network structure that can require coordination to form is balance. In a signed network, a triangle is (classically) balanced when the product of the positive $(+)$ and negative (-) ties is positive (Cartwright \& Harary, 1956). More conceptually, balance occurs when actors form positive and negative ties in patterns that obey a set of aphoristic rules such as "a friend of a friend is a friend" and "an enemy of an enemy is a friend" (Doreian et al., 1996, p. 115) that find their origins in the sociology of Simmel and psychology of Heider (Krackhardt \& Handcock, 2006). In a dynamic context, balance increases locally as actors re-arrange their social ties to achieve greater balance in their own social circles, and globally because unbalanced triads are inherently unstable (Doreian et al., 1996). The effects of tendencies toward balance are reflected in static networks when a large fraction of signed triangles are balanced (i.e., a high triangle index; Aref \& Wilson, 2018). To date, few studies have investigated balance in preschoolers' networks mainly because signed networks are challenging to collect from this population. Daniel et al. (2016) did not find evidence of balance in preschoolers' self-reported affect toward others. However, this study focused on psychological balance, whereas our focus is on balance in social interactions. Again, because social play involves more coordination than parallel play, if backbone models validly infer young children's networks, we would expect networks inferred from social play to exhibit more balance than networks inferred from parallel play.

\section{Method}

\subsection{Setting and Sample}

To provide evidence of the validity of bipartite projection backbone models for inferring young children's signed networks, we used observational data collected in one 3-year-old and one 4-year-old preschool classroom. Both classrooms were part of a university preschool located in the U.S. Midwest that serves children and families from the surrounding community. Children in these classrooms attended preschool in the morning between 8:30 and 11:30am and received a curriculum that included both structured activities with teachers as well as periods of unstructured free play. During free play periods, children chose their own activities and playmates. Both classrooms included roughly equal numbers of students (i.e., 3-year-old classroom $N=17$, 4-year-old classroom $N=18$ ). Demographics for children in each classroom including gender, race/ethnicity, and age are provided in Table 1 .

Table 1: Sample Demographics \& Observations

\begin{tabular}{lll}
\hline & $\begin{array}{l}\text { 3-year-old } \\
(\mathrm{N}=17)\end{array}$ & $\begin{array}{l}\text { 4-year-old } \\
(\mathrm{N}=18)\end{array}$ \\
\hline Gender & $8(47.06 \%)$ & $9(50 \%)$ \\
Boy & $9(52.94 \%)$ & $9(50 \%)$ \\
Girl & & \\
& & \\
Race/Ethnicity & $3(17.65 \%)$ & $0(0 \%)$ \\
Asian & $3(17.65 \%)$ & $0(0 \%)$ \\
Black/African American & $8(47.06 \%)$ & $14(77.78 \%)$ \\
White & $1(5.88 \%)$ & $1(5.55 \%)$ \\
Multi-racial/Multi-ethnic & $2(11.76 \%)$ & $3(16.67 \%)$ \\
Missing & & \\
Age (in months), M(SD) & $42.35(4.74)$ & $51.78(3.57)$ \\
Observations & & \\
Social Play & & 994 \\
Parallel Play & 668 & 423 \\
\hline
\end{tabular}

\subsection{Procedure}

Between October 2012 and May 2013, we collected data on children's gender from preschool staff 
and data on parallel play and social play interactions using scan observational procedures similar to those applied in previous preschool studies (e.g., Daniel et al., 2019; Hanish et al., 2005; Schaefer et al., 2010). During each classroom observational period, an observer received a randomly ordered list of children and pictures identifying each child in the classroom. The observer rotated through the list, observing each child for a period of 10 seconds and recording their most dominant behavior from a list of 7 behaviors (i.e., parallel play, social play rough and tumble play, onlooking behavior, solitary play, unoccupied behavior, and teacher-oriented behavior). When a behavior involved interactions with peers (e.g., parallel play, social play), the observer also coded the identities of all peers engaged in the interaction.

A team of trained observers conducted observations on Mondays through Thursdays. Observations were scheduled during periods of unstructured free play to ensure that observations reflected children's autonomous choice of playmates. In the 3year-old classroom, observers collected a total of 1102 scan observations involving parallel play $(n=$ $434)$ or social play $(n=668)$. In the 4 -year-old classroom, observers collected a total of 1417 scan observations involving parallel play $(n=423)$ or social play $(n=994)$. In addition to these 2519 observations which we use to infer children's networks with methods described below, pairs of observers also recorded observations of the same focal child 216 times to evaluate the reliability of these observations. In these reliability-check observations, observers agreed on the observed behavior $79.89 \%$ of the time, and on the exact set of peers involved in social or parallel play $81.94 \%$ of the time.

All procedures were approved by the authors' institutional review board (Protocol 11-1198). Because observations of classroom social interactions posed minimal risk to children and could not be conducted without observing all children in the classroom, we received a waiver of active parental consent and student assent (Klovdahl, 2005). This ensured that we were able to collect data on all children and all social interactions in each classroom.

\subsection{Signed network inference}

Within each classroom, we organized the scan observation data in a matrix $\mathbf{M}$, which represents a bipartite network where $M_{i k}=1$ if child $i$ was observed participating in a play event $k$. From this matrix, a weighted bipartite projection $\mathbf{P}$ can be constructed as $\mathbf{P}=\mathbf{M M}^{\prime}$, where $P_{i j}$ records the number of times children $i$ and $j$ were observed playing together. Such a co-playing network is not very useful because nearly every pair of children was observed playing together at least a few times. Bipartite projection backbone models provide a test for determining whether the number of times two children were observed playing together (i.e., $P_{i j}$ ) was statistically significantly larger than expected at random and therefore suggestive of a positive social tie, or was statistically significantly smaller than expected and therefore suggestive of a negative social tie.

Many different bipartite projection backbone models are available, but here we used the fixed degree sequence model (FDSM) because it is more statistically powerful than the alternatives (Neal et al., 2021). The mathematical details of the FDSM have been extensively described elsewhere (e.g., Gotelli, 2000; Zweig \& Kaufmann, 2011; Neal et al., 2021), so here we briefly outline the process. A Monte Carlo method was required to determine whether $P_{i j}$ is statistically significantly large or small. First, a random bipartite matrix $\mathbf{M}^{*}$ was constructed that has the same row and column sums as M. Second, a random projection $\mathbf{P}^{*}$ was constructed from $\mathbf{M}^{*}$ as $\mathbf{P}^{*}=\mathbf{M}^{*} \mathbf{M}^{* \prime}$. This process was repeated many times to obtain a distribution of $P_{i j}^{*}$, to which $P_{i j}$ was compared using a chosen level of statistical significance $(\alpha)$. Applying this statistical test to each dyad, a signed network $\mathbf{S}$ was inferred such that

$S_{i j}= \begin{cases}1 \text { (i.e., positive tie) } & \text { if } \operatorname{Pr}\left(P_{i j}^{*} \geq P_{i j}\right)<\frac{\alpha}{2}, \\ -1 \text { (i.e., negative tie) } & \text { if } \operatorname{Pr}\left(P_{i j}^{*} \leq P_{i j}\right)<\frac{\alpha}{2}, \\ 0 \text { (i.e., no tie) } & \text { otherwise. }\end{cases}$

We used a conventional $\alpha=0.05$ significance level to infer whether a given dyad had a positive tie, a negative tie, or no tie. In practice, this required computing $\operatorname{Pr}\left(P_{i j}^{*} \geq P_{i j}\right)$ and $\operatorname{Pr}\left(P_{i j}^{*} \geq P_{i j}\right)$ with sufficient precision to be confident in any decisions that it is smaller than 0.025 (i.e., $\alpha / 2$ ). Using the estimation provided by Neal et al. (2021), we found that making such decisions with 5\% Type-I error and 5\% Type-II error (i.e., 95\% Power) required $\approx 165,000$ trials. This highlights a key practical challenge to using FDSM: efficiently generating $\mathbf{M}^{*}$ such a large number of times. To overcome this challenge, we used the 'fastball' algorithm, which has been proven to randomly sample $\mathbf{M}^{*}$ from the space of all binary matrices with given 
row and column sums (Godard \& Neal, 2021).

The FDSM is a generic model for inferring a signed (or binary) unipartite network from a weighted bipartite projection. When applied in this context, it allowed us to infer a signed network of social ties among preschool children from their observed play behaviors. These inferences depend on whether the number of times two children were observed playing together was significantly larger (a positive tie is inferred) or smaller (a negative tie is inferred) than the number of times they would be observed playing together if they selected play partners randomly. However, rather than simply judging whether the number of co-playing observations was large or small in absolute terms, the FDSM allowed us to take into account the fact that children differed in how often they play with others (i.e., sociability), and play groups differed in their numbers of participants. For each classroom, we used the FDSM to infer a network from all social and parallel play observations, which we used to test hypotheses concerning gender homophily. We then used it to infer separate networks from social play behaviors and from parallel play behaviors, which we used to test hypotheses concerning triadic closure and structural balance. All data and code necessary to replicate these analyses is available at https://osf.io/q7nh6.

\section{Results}

\subsection{Inferred signed networks}

To illustrate the types of signed networks this approach is able to infer from scan observation data, Figure 1 shows the network inferred from all social and parallel play observations in the 3-year-old classroom, and Figure 2 shows the network inferred in the 4-year-old classroom. Pink circles represent girls and blue circles represent boys. Solid green lines represent pairs of children who played together statistically significantly more than expected at random, which we treated as positive ties. There were 29 positive ties in the 3 -year-old classroom and 31 positive ties in the 4 -year-old classroom. In contrast, dashed red lines represent pairs of children who played together statistically significantly less than expected at random, which we treated as negative ties. There were 38 negative ties in the 3 -year-old classroom and 54 negative ties in the 4year-old classroom. Both sociograms were drawn by applying the Kamada \& Kawai (1989) layout to the positive edges only.

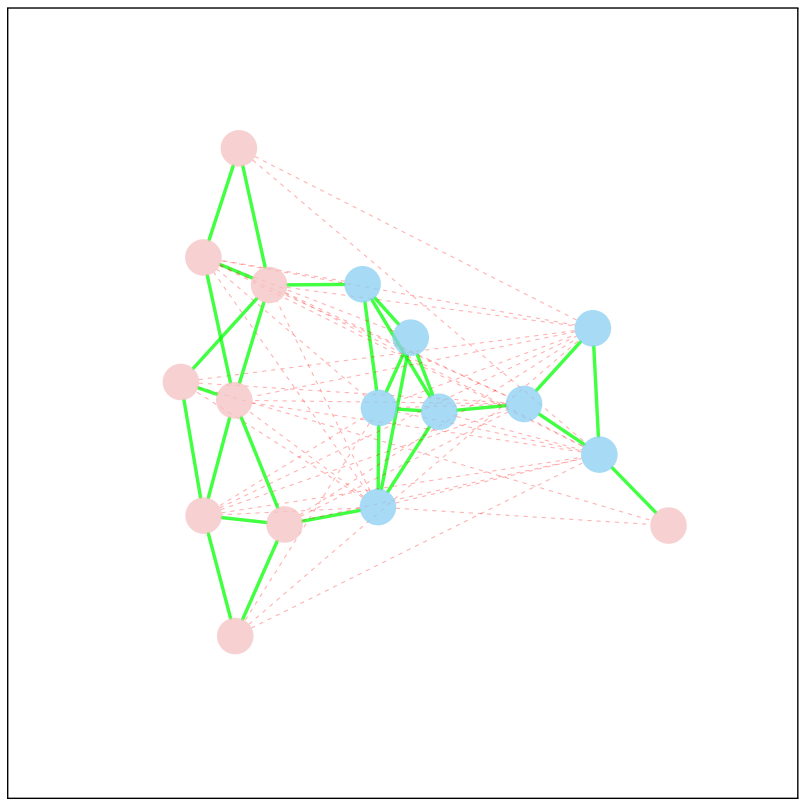

Figure 1: Signed network inferred from observed play behaviors of 3-year-old girls (pink) and boys (blue). Solid green lines represent positive ties, while dashed red lines represent negative ties.

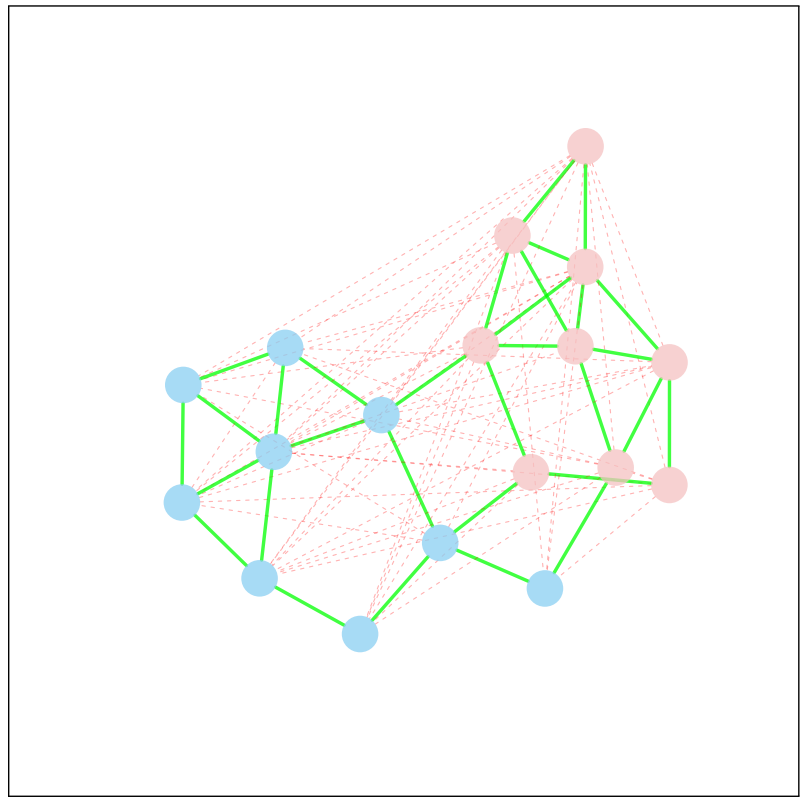

Figure 2: Signed network inferred from observed play behaviors of 4-year-old girls (pink) and boys (blue). Solid green lines represent positive ties, while dashed red lines represent negative ties. 
Table 2: Gender homophily

\begin{tabular}{l|cc|cc}
\hline & \multicolumn{2}{|c|}{ 3-year-old dyads } & \multicolumn{2}{|c}{ 4-year-old dyads } \\
\hline Tie & $\underline{\text { Different }}$ & $\underline{\text { Same }}$ & $\underline{\text { Different }}$ & $\underline{\text { Same }}$ \\
Positive & $3(10.3 \%)$ & $26(89.7 \%)$ & $3(9.7 \%)$ & $28(90.3 \%)$ \\
Negative & $32(84.2 \%)$ & $6(15.8 \%)$ & $45(83.3 \%)$ & $9(16.7 \%)$ \\
None & $37(53.6 \%)$ & $32(46.4 \%)$ & $33(48.5 \%)$ & $35(51.5 \%)$ \\
\hline & \multicolumn{3}{|l|}{ Fisher's exact $p<0.001$} & \multicolumn{2}{|c|}{ Fisher's exact $p<0.001$} \\
\hline
\end{tabular}

\begin{tabular}{|c|c|c|c|c|}
\hline & \multicolumn{2}{|c|}{ 3-year-old networks } & \multicolumn{2}{|c|}{ 4-year-old networks } \\
\hline Triad & Parallel Play & Social Play & Parallel Play & Social Play \\
\hline$\overline{\text { Closed }}$ & $15(35.7 \%)$ & $30(50 \%)$ & $6(16.2 \%)$ & $18(24.7 \%)$ \\
\hline Open & $27(64.3 \%)$ & $30(50 \%)$ & $31(83.8 \%)$ & $55(75.3 \%)$ \\
\hline & Fisher's exa & $p=0.1634$ & Fisher's ex & $p=0.342$ \\
\hline
\end{tabular}

\subsection{Gender homophily}

To provide evidence of the validity of our inferred networks, we first examined patterns of gender homophily and heterophily in the classroom networks inferred from all social and parallel play observations. If the backbone model validly inferred young children's signed social ties, then positive ties should occur more often in same-gender dyads, while negative ties should occur more often in different-gender dyads. These patterns of gender homophily and heterophily are clearly visible in both Figure 1 and Figure 2. They are corroborated in cross-tabulations reported in Table 2, which examined the relationship between the type of tie (i.e., positive, negative vs. none) and the gender composition of peer dyads (i.e., different-gender vs. same-gender). As expected, the majority of positive ties occurred in same-gender dyads in both the 3 -year-old classroom $(89.7 \%)$ and the 4 -year-old classroom (90.3\%). Likewise, the majority of negative ties occurred in different-gender dyads in both the 3 -year-old classroom $(84.2 \%)$ and the 4 -yearold classroom $(83.3 \%)$. These patterns of gender homophily in positive ties and gender heterophily in negative ties were statistically significant in both classrooms (Fisher's exact test $p<0.001$ ).

One risk is that the inferred networks exhibited these expected gender homophily patterns because the inferences were simply driven by gender, such that most same-gender dyads were inferred to have a positive tie and most different-gender dyads were inferred to have a negative tie. However, the results in Table 2 demonstrate that this was not happening. Specifically, we observed that the backbone model frequently inferred that no tie exists in a dyad, and that it was equally likely to infer that no tie existed in same-gender and in different-gender dyads (46.4\% vs. $53.6 \%$ among 3 -year olds; $51.5 \%$ vs. $48.5 \%$ among 4 -year-olds). This pattern indicates that backbone model inferences were able to distinguish same-gender dyads that have a positive tie from those that have no tie, and likewise to distinguish different-gender dyads that have a negative tie from those that have no tie.

\subsection{Triadic closure and structural balance}

Using separate networks inferred from social play behaviors and from parallel play behaviors, we examined triadic closure. If the backbone model validly inferred young children's signed ties, then networks inferred from social play should exhibit more transitivity in positive ties than networks inferred from parallel play. Table 3 reports, for each classroom, the relationship between the type of network (i.e., parallel play vs. social play) and the type of triad (i.e., closed vs. open; using positive ties only). In these cross-tabulations, the reported percentage of closed triads reflects each network's transitivity. As expected, networks inferred from social play exhibited more transitivity than networks inferred from parallel play among 3-year-olds ( $50 \%$ for social play vs. $35.7 \%$ for parallel play) and among 4-year-olds (24.7\% for social play vs. $16.2 \%$ for parallel play). However, in part due to the small cell counts, these differences were not statistically significantly different (Fisher's exact $p=0.1634$ and $p=0.342)$.

Again using separate networks inferred from social play behaviors and from parallel play behaviors, we also examined balance. If the backbone 
Table 4: Structural balance

\begin{tabular}{|c|c|c|c|c|}
\hline & \multicolumn{2}{|c|}{ 3-year-old networks } & \multicolumn{2}{|c|}{ 4-year-old networks } \\
\hline Triad & Parallel Play & Social Play & Parallel Play & Social Play \\
\hline Balanced & $124(77.5 \%)$ & $244(92.4 \%)$ & $64(76.2 \%)$ & $452(86.3 \%)$ \\
\hline Unbalanced & $36(22.5 \%)$ & $20(7.6 \%)$ & $20(23.8 \%)$ & $72(13.7 \%)$ \\
\hline & \multicolumn{2}{|c|}{ Fisher's exact $p<0.001$} & \multicolumn{2}{|c|}{ Fisher's exact $p=0.02156$} \\
\hline
\end{tabular}

model validly inferred young children's networks, then networks inferred from social play should exhibit more balance than networks inferred from parallel play. Table 4 reports, for each classroom, the relationship between the type of network (i.e., parallel play vs. social play) and the type of triad (i.e., balanced vs. unbalanced). In these crosstabulations, the reported percentage of balanced triads reflects each network's triangle index, and thus its degree of classical balance. As expected, networks inferred from social play exhibited significantly more balance than networks inferred from parallel play among 3-year-olds (i.e., $92.4 \%$ for social play vs. $77.5 \%$ for parallel play, Fisher's exact $p<0.001)$ and among 4-year-olds $(86.3 \%$ for social play vs. $76.2 \%$ for parallel play, Fisher's exact $p=0.02156)$.

\section{Discussion}

Although early childhood is a critical developmental period for the formation of social ties (e.g., Daniel et al., 2016; Irwin et al., 2021; Martin et al., 2005; Schaefer et al., 2010), studies of preschoolers' networks remain uncommon, in part, due to measurement challenges (Neal, 2020). In this paper, we explored the use of a bipartite projection backbone model - the fixed degree sequence model - to infer positive and negative ties among preschoolers from observations of their play interactions. Using observational data from two preschool classrooms, we offered evidence of the validity of the inferred networks by demonstrating that they exhibit structural patterns that are consistent with existing theoretical and empirical expectations in the early childhood literature.

Using networks inferred from all social and parallel play observations, we found evidence of gender homophily in positive ties and gender heterophily in negative ties. These findings are consistent with gender schema theory which suggests that preschoolers form gender-based assumptions that prompt preferences for same gender play- mates (Martin \& Halverson, 1981; Martin \& Ruble, 2004). They are also consistent with welldocumented empirical evidence that preschool children tend to form positive ties with same gender peers (e.g., Chen et al., 2020; Daniel et al., 2016; Martin et al., 2013; Schaefer et al., 2010) and negative ties with peers of different genders (Daniel et al., 2016; Van den Oord et al., 2000). Additionally, using separate networks inferred from social play behaviors and from parallel play behaviors, we found networks inferred from social play exhibited more closed and balanced triads than networks inferred from parallel play. These findings are consistent with theoretical expectations that networks derived from social play, which includes active interactions, should contain more structures that suggest peer coordination than parallel play, which only includes passive interactions (Coplan \& Arbeau, 2009; Parten, 1932).

As a set, our findings provide evidence of the validity of backbone models for inferring signed networks from play observations in early childhood. These models have benefits for measuring and understanding young children's social ties. First, developmental researchers have struggled with establishing criteria for inferring positive ties from children's play observations. As Daniel et al. (2013) noted, inferring positive ties from observational data "involves some degree of arbitrariness and at the moment there is not a generally accepted approach for doing so" (p. 26). Backbone models reduce this arbitrariness because they provide a formal statistical test of whether the number of times two children were observed playing together was significantly larger than expected at random. Second, negative ties are particularly challenging to measure among young children because they are low rate and difficult to collect via self-report or directly observe (Roseth et al., 2008; Vaughn et al., 2003). Backbone models provide a method of inferring negative ties because they provide a formal statistical test of whether the number of times two children were observed playing together was signif- 
icantly smaller than expected at random. Thus, bipartite projection backbone models open new possibilities for developmental researchers to examine signed networks in early childhood. Although we have focused on preschool children, these methods may also open similar possibilities for other populations from whom self-report networks are challenging to collect (e.g., adults with developmental disabilities or memory impairments).

Our results provide initial evidence of the validity of backbone models for inferring signed networks among young children but should be interpreted in light of some limitations. Because collecting observational data is resource intensive, we were only able to examine evidence for the validity of networks inferred in two classrooms at a Midwestern US university preschool. Future work is needed to determine whether these findings would generalize to different preschool settings and demographically and geographically diverse samples. Additionally, we were unable to collect self-report data on children's positive and negative ties. Future work could add to our evidence of validity by collecting both observational and self-report data, then examining whether networks inferred from observations resemble those reported by children.

This analysis also highlights some potential limitations of the backbone models themselves. First, these models require a large number of observations from which to infer social ties. In our case, we successfully inferred a network from as few as 423 observations (i.e., observations of parallel play in the 4-year-old classroom). However, conducting this number of observations may be impractical in some settings. Second, the fixed degree sequence model is computationally costly. In our case, 165,000 Monte Carlo trials were necessary, which required less than a minute. However, inferring networks using a more conservative significance level (i.e., $\alpha<0.05$ ) would require more trials, and inferring networks from larger numbers of observations would require more time per trial. Alternative backbone models such as the stochastic degree sequence model are more efficient, however this efficiency comes at the cost of reduced statistical power Neal et al. (2021).

These limitations notwithstanding, we have presented preliminary evidence of the validity of early childhood networks inferred from play observations using backbone models. This approach offers new opportunities for studying networks during the developmentally formative period of early childhood, and for understanding the role of both positive and negative ties on a range of social, cognitive, and health outcomes.

\section{Data availability statement}

All data and code necessary to replicate this study are available at https://osf.io/q7nh6.

\section{Contributor statement}

JWN and CED collected the data, ZPN analyzed the data, JWN and ZPN drafted the manuscript, all authors revised the manuscript.

\section{Disclosures}

The authors declare no conflicts of interest.

\section{References}

adams, j. (2020). Gathering social network data. SAGE Publications Incorporated.

Aref, S., \& Wilson, M. C. (2018). Measuring partial balance in signed networks. Journal of Complex Networks, 6 , 566-595.

Cartwright, D., \& Harary, F. (1956). Structural balance: a generalization of heider's theory. Psychological review, $63,277$.

Chen, J., Justice, L. M., Rhoad-Drogalis, A., Lin, T.-J., \& Sawyer, B. (2020). Social networks of children with developmental language disorder in inclusive preschool programs. Child development, 91, 471-487.

Coplan, R. J., \& Arbeau, K. A. (2009). Peer interactions and play in early childhood. In K. Rubin, W. Bukowski, \& B. E. Laursen (Eds.), Social, Emotional, and Personality Development in Context. Handbook of Peer Interactions, Relationships, and Groups (p. 143-161). The Guilford Press.

Cronbach, L. J., \& Meehl, P. E. (1955). Construct validity in psychological tests. Psychological bulletin, 52, 281.

Daniel, J. R., Santos, A. J., Antunes, M., Fernandes, M., \& Vaughn, B. E. (2016). Co-evolution of friendships and antipathies: a longitudinal study of preschool peer groups. Frontiers in Psychology, 7, 1509.

Daniel, J. R., Santos, A. J., Fernandes, C., \& Vaughn, B. E. (2019). Network dynamics of affiliative ties in preschool peer groups. Social Networks, 57, 63-69.

Daniel, J. R., Santos, A. J., Peceguina, I., \& Vaughn, B. E. (2013). Exponential random graph models of preschool affiliative networks. Social Networks, 35, 25-30.

DeLay, D., Hanish, L. D., Martin, C. L., \& Fabes, R. A. (2016). Peer effects on head start children's preschool competency. Developmental Psychology, 52, 58.

Doreian, P., Kapuscinski, R., Krackhardt, D., \& Szczypula, J. (1996). A brief history of balance through time. Journal of Mathematical Sociology, 21, 113-131.

Goble, P., Martin, C. L., Hanish, L. D., \& Fabes, R. A. (2012). Children's gender-typed activity choices across preschool social contexts. Sex Roles, 67, 435-451. 
Godard, K., \& Neal, Z. P. (2021). fastball: A fast algorithm to sample binary matrices with fixed marginals. arXiv preprint arXiv:2112.04017,

Gotelli, N. J. (2000). Null model analysis of species cooccurrence patterns. Ecology, 81, 2606-2621.

Granovetter, M. S. (1973). The strength of weak ties. American journal of sociology, 78, 1360-1380.

Göncü, A., Patt, M. B., \& Kouba, E. (2002). Understanding young children's pretend play in context. In P. K. Smith, \& C. H. E. Hart (Eds.), Blackwell Handbook of Childhood Social Development (p. 418-437). Blackwell.

Hanish, L. D., Martin, C. L., Fabes, R. A., Leonard, S., \& Herzog, M. (2005). Exposure to externalizing peers in early childhood: Homophily and peer contagion processes. Journal of abnormal child psychology, 33, 267-281.

Irwin, V., Zhang, J., Wang, K., Roberts, A., York, C. Barmer, A., Bullock Mann, F., Dilig, R., \& Parker, S. (2021). The condition of education 2021. U.S. Department of Education. Washington, DC: National Center for Education Statistics, .

Kamada, T., \& Kawai, S. (1989). An algorithm for drawing general undirected graphs. Information processing letters, $31,7-15$.

Klovdahl, A. S. (2005). Social network research and human subjects protection: Towards more effective infectious disease control. Social Networks, 27, 119-137.

Krackhardt, D., \& Handcock, M. S. (2006). Heider vs simmel: Emergent features in dynamic structures. In $I C M L$ Workshop on Statistical Network Analysis (pp. 14-27). Springer.

La Freniere, P., Strayer, F. F., \& Gauthier, R. (1984) The emergence of same-sex affiliative preferences among preschool peers: A developmental/ethological perspective. Child development, (pp. 1958-1965).

Luckey, A. J., \& Fabes, R. A. (2005). Understanding nonsocial play in early childhood. Early Childhood Education Journal, 33, 67-72

Maccoby, E. E. (1990). Gender and relationships: A developmental account. American psychologist, 45, 513.

Maccoby, E. E., \& Jacklin, C. N. (1987). Gender segregation in childhood. In Advances in child development and behavior (pp. 239-287). Elsevier volume 20.

Martin, C. L., \& Fabes, R. A. (2001). The stability and consequences of young children's same-sex peer interactions. Developmental psychology, 37, 431.

Martin, C. L., Fabes, R. A., Hanish, L., Leonard, S., \& Dinella, L. M. (2011). Experienced and expected similarity to same-gender peers: Moving toward a comprehensive model of gender segregation. Sex roles, 65, 421-434.

Martin, C. L., Fabes, R. A., Hanish, L. D., \& Hollenstein, T. (2005). Social dynamics in the preschool. Developmental Review, 25, 299-327.

Martin, C. L., \& Halverson, C. F. (1981). A schematic processing model of sex typing and stereotyping in children. Child development, (pp. 1119-1134).

Martin, C. L., Kornienko, O., Schaefer, D. R., Hanish, L. D. Fabes, R. A., \& Goble, P. (2013). The role of sex of peers and gender-typed activities in young children's peer affiliative networks: A longitudinal analysis of selection and influence. Child development, 84, 921-937.

Martin, C. L., \& Ruble, D. (2004). Children's search for gender cues: Cognitive perspectives on gender development. Current directions in psychological science, 13, 67-70.

Martin, C. L., \& Ruble, D. N. (2010). Patterns of gender development. Annual review of psychology, 61, 353-381.
McCandless, B. R., \& Marshall, H. R. (1957). A picture sociometric technique for preschool children and its relation to teacher judgments of friendship. Child development, (pp. 139-147).

Messick, S. (1995). Validity of psychological assessment: Validation of inferences from persons' responses and performances as scientific inquiry into score meaning. American psychologist, 50, 741 .

Neal, J. W. (2020). A systematic review of social network methods in high impact developmental psychology journals. Social Development, 29, 923-944.

Neal, J. W., Durbin, C. E., Gornik, A. E., \& Lo, S. L. (2017). Codevelopment of preschoolers' temperament traits and social play networks over an entire school year. Journal of Personality and Social Psychology, 113, 627 - 640 .

Neal, Z. P. (2014). The backbone of bipartite projections: Inferring relationships from co-authorship, co-sponsorship, co-attendance and other co-behaviors. Social Networks, 39, 84-97.

Neal, Z. P., Domagalski, R., \& Sagan, B. (2021). Comparing alternatives to the fixed degree sequence model for extracting the backbone of bipartite projections. Scientific reports, $11,1-13$. doi:10.1038/s41598-021-03238-3.

Van den Oord, E. J., Rispens, J., Goudena, P. P., \& Vermande, M. (2000). Some developmental implications of structural aspects of preschoolers' relations with classmates. Journal of Applied Developmental Psychology, 21, 619-639.

Ostrov, J. M., \& Keating, C. F. (2004). Gender differences in preschool aggression during free play and structured interactions: An observational study. Social development, 13, 255-277.

Parten, M. B. (1932). Social participation among pre-school children. The Journal of Abnormal and Social Psychology, 27, 243.

Roseth, C. J., Pellegrini, A. D., Dupuis, D. N., Bohn, C. M., Hickey, M. C., Hilk, C. L., \& Peshkam, A. (2008). Teacher intervention and us preschoolers' natural conflict resolution after aggressive competition. Behaviour, (pp. 16011626).

Schaefer, D. R., Light, J. M., Fabes, R. A., Hanish, L. D., \& Martin, C. L. (2010). Fundamental principles of network formation among preschool children. Social Networks, 32, 61-71.

Strayer, F. F., \& Santos, A. J. (1996). Affiliative structures in preschool peer groups. Social Development, 5, 117-130.

Vaughn, B. E., Vollenweider, M., Bost, K. K., Azria-Evans, M. R., \& Snider, J. B. (2003). Negative interactions and social competence for preschool children in two samples: Reconsidering the interpretation of aggressive behavior for young children. Merrill-Palmer Quarterly (1982-), (pp. 245-278).

Zweig, K. A., \& Kaufmann, M. (2011). A systematic approach to the one-mode projection of bipartite graphs. Social Network Analysis and Mining, 1, 187-218. 